\title{
SOME REMARKS ON THE AVERAGE RANGE OF A VECTOR MEASURE
}

\author{
FRANCISCO J. FRENICHE
}

(Communicated by R. Daniel Mauldin)

\begin{abstract}
We study some conditions on the average range of a vector measure with values in the bidual of a Banach space which imply that the range is contained in the space. We prove that Geitz's condition is a sufficient one if the dual closed unit ball is weak-star sequentially compact. We also show how to reduce to measures with values in the bidual of $l^{\infty}$.
\end{abstract}

In this paper we consider the following question: given a vector measure $\nu$ with values in the bidual $X^{* *}$ of the Banach space $X$, under what conditions can we say that $\nu$ actually takes its values inside $X$ ? We shall also assume that $\nu$ is defined on a probability space $(S, \Sigma, \mu)$ and that it is absolutely continuous with respect to $\mu$. This problem is connected with the following ones.

(a) Let $\left(f_{n}\right)$ be an equi-integrable sequence of functions, bounded in $L^{1}$ norm; when is $\left(f_{n}\right)$ weakly null in $L^{1}(\mu)$ ? This problem is equivalent to the former one when $X=c_{0}$ so that $X^{* *}=l^{\infty}$. Indeed, it suffices to look at the vector measure given by

$$
\nu(A)=\left(\int_{A} f_{n} d \mu\right)
$$

and to apply Dunford-Pettis's characterization of the weakly null sequences in $L^{\prime}(\mu)[4]$.

(b) Let $f: S \rightarrow X$ be a bounded and scalarly measurable function. The indefinite Dunford integral $\nu$ of $f$ is defined by

$$
\left\langle\nu(A), x^{*}\right\rangle=\int_{A}\left\langle x^{*}, f(s)\right\rangle d \mu(s)
$$

for $A \in \Sigma$ and $x^{*} \in X^{*}$; when is $\nu(A) \in X$ for any measurable $A$ ?, that is, when is $f$ Pettis integrable? [3].

There are a lot of easy conditions which imply that the range of $\nu$ is contained in $X$. Let us assume for instance that for every $A \in \Sigma^{+}$-that is, $A \in \Sigma$ and $\mu(A)>0$-there exists $B \in \Sigma_{A}^{+}$-that is, $B \in \Sigma^{+}$and $B \subset A$-so that

Received by the editors July 25,1988 .

1980 Mathematics Subject Classification (1985 Revision). Primary 28B05, 46G10.

Key words and phrases. Weakly convergent sequences in $L^{1}$, Pettis integral, core of a vector measure. 
$\nu(B) \in X$; then an exhaustion argument gives $\nu(\Sigma) \subset X$. Another sufficient condition is the following one: $\nu(A) \in X$ for $A$ running in a generating subalgebra of $\Sigma$; then the continuity of $\nu$ when regarded as a function on the pseudometric space associated to $\mu$, as well as the density of the elements of that subalgebra, allow us to conclude that $X$ contains the range of $\nu$.

We are interested in conditions on the average range

$$
\mathscr{A}_{\nu}(A)=\left\{\frac{\nu(B)}{\mu(B)}: B \in \Sigma_{A}^{+}\right\}
$$

of the vector measure which guarantee that its range is contained in $X$. We recall Geitz's condition for Pettis integrability: Geitz defines in [5] the corc of $f$ on $A$ by

$$
\text { core }_{f}(A)=\cap\{\text { weak-star closure of } \operatorname{co} f(A \backslash B): \mu(B)=0\}
$$

which, as noticed in [7], can be expressed in terms of the average range of $\nu$, the indefinite Dunford integral of $f$, namely

$$
\operatorname{core}_{\nu}(A)=\text { weak-star closure of } \operatorname{co}\left(\mathscr{A}_{\nu}(A)\right) \text {. }
$$

The condition of Geitz reads as follows

$$
\text { core }_{\nu}(A) \cap X \neq \varnothing
$$

for every $A \in \Sigma^{+}$.

The next theorem answers the question when the Banach space $X$ has Mazur property, in particular, when $X$ is a weakly compact generated Banach space [2]. Thus a sufficient condition for question (a) is Geitz's one.

Theorem 1. Let $\nu: \Sigma \rightarrow X^{* *}$ be an absolutely continuous vector measure satisfying Geitz's condition. Then $\nu(A)$ is weak-star sequentially continuous, for every $A \in \Sigma$.

Proof. Assume, by contradiction, that there is $B \in \Sigma$ and a weak-star null sequence $\left(x_{n}^{*}\right)$ so that $\left\langle\nu(B), x_{n}^{*}\right\rangle \geq 2$ for every $n$.

Let $f_{n}$ be the Radon-Nikodym derivative of the scalar measure $x_{n}^{*} \circ \nu$ with respect to $\mu$. As $\left(x_{n}^{*}\right)$ is bounded in $X$ and $\nu$ is absolutely continuous with respect to $\mu$, the sequence $\left(f_{n}\right)$ is equi-integrable and bounded in $L^{1}(\mu)$, so we can assume without lost of generality that $\left(f_{n}\right)$ is weakly converging in $L^{1}(\mu)$ to a function $f$.

As the weak- and norm-closure are the same for convex sets, we can construct a sequence $\left(J_{n}\right)$ with $J_{n} \subset \mathbf{N}$ finite, $\max J_{n}<\min J_{n+1}$, and a sequence $\left(F_{n}\right)$ with $F_{n} \in \operatorname{co}\left(\left\{f_{k}: k \in J_{n}\right\}\right)$ satisfying $\left\|F_{n}-f\right\|_{1} \rightarrow 0$. By passing to a subsequence if necessary, we can also assume that $\left(F_{n}\right)$ converges to $f$ almost everywhere, hence by Egorov's theorem, that $F_{n} \rightarrow f$ almost uniformly on $S$.

On the other hand we can choose $B_{1} \in \Sigma_{B}^{+}$so that $f \geq 1$ on $B_{1}$ almost everywhere. Indeed, it suffices to notice that $\mu(S)=1$ and $\int_{B} f d \mu \geq 2$.

Let $A \in \Sigma^{+}$so that $A \subset B_{1}$ and $F_{n} \rightarrow f$ uniformly on $A$. Notice that we also have $f \geq 1$ almost everywhere on $A$. 
We claim that $X \cap \operatorname{core}_{\nu}(A)=\varnothing$. To see this, let us fix $x \in X$. Then we can choose $n$ so that $\left\langle x_{k}^{*}, x\right\rangle \leq \frac{1}{6}$ for $k \geq n$, and $m$ so that $\min J_{m} \geq n$ and $\left|F_{m}(s)-f(s)\right| \leq \frac{1}{3}$, for every $s \in A$.

Let $x^{*}$ be the convex combination of $\left\{x_{k}^{*}: k \in J_{m}\right\}$ constructed with the same weights as $F_{m}$ when we write it in terms of $\left\{f_{k}: k \in J_{m}\right\}$. Then we have $\left\langle x^{*}, x\right\rangle \leq \frac{1}{6}$.

At the same time,

$$
\left\langle\nu(C), x^{*}\right\rangle=\int_{C} F_{m} d \mu \geq \int_{C} f d \mu-\frac{1}{3} \mu(C)
$$

and so

$$
\left\langle\nu(C), x^{*}\right\rangle \geq \frac{2}{3} \mu(C)
$$

for every $C \in \Sigma_{A}^{+}$.

Therefore we have $x^{*} \geq \frac{2}{3}$ on $\mathscr{A}_{\nu}(A)$, so that $x \notin \operatorname{core}_{\nu}(A)$.

Our next theorem provides a complete answer for a certain class of Banach spaces. In its proof we use Theorem 1.

Theorem 2. Let $X$ be a Banach space such that its dual closed unit ball is weakstar sequentially compact. If the measure $\nu$ satisfies Geitz's condition, then its range is contained in $X$.

Proof. By Grothendieck's completeness theorem, if $\nu(B) \notin X$ then there is $\varepsilon>0$ such that for any $Y \subset X$ finite, there exists $x^{*} \in X^{*}$ with $\left\|x^{*}\right\| \leq 1$, $\left|\left\langle x^{*}, x\right\rangle\right| \leq 1$ for $x \in Y$, and $\left\langle\nu(B), x^{*}\right\rangle \geq \varepsilon$. let

Let $f_{x^{*}}$ be the Radon-Nikodym derivative of $x^{*} \circ \nu$ with respect to $\mu$, and

$$
\mathscr{B}_{Y}=\left\{f_{x^{*}}:\left\|x^{*}\right\| \leq 1,\left\langle\nu(B), x^{*}\right\rangle \geq \varepsilon \text { and }\left|\left\langle x^{*}, x\right\rangle\right| \leq 1 \text { for } x \in Y\right\} .
$$

By weak compactness, we can choose $f$ in the weak closure of $\mathscr{B}_{Y}$ in $L^{1}(\mu)$, for every $Y \subset X$ finite. As the sets $\mathscr{B}_{Y}$ are convex, we get that $f$ is in the norm closure of $\mathscr{B}_{Y}$ in $L^{1}(\mu)$, for every such $Y$.

Since $\int_{B} f d \mu \geq \varepsilon$ and $\mu(S)=1$, we have $f \geq \varepsilon / 2$ almost everywhere on some $A \in \Sigma_{B}^{+}$.

We now show that core $_{\nu}(A)$ and $X$ are disjoint sets, contradicting the hypothesis of the theorem. Let $x \in X$ be given.

Then $f \in \mathscr{B}_{\{n x\}}$ for every $n$, and so we can choose a sequence $\left(x_{n}^{*}\right)$ which satisfies $\left\|x_{n}^{*}\right\| \leq 1,\left\langle x_{n}^{*}, x\right\rangle \leq n^{-1}$ and, if $f_{n}$ is the density of $x_{n}^{*} \circ \nu,\left(f_{n}\right)$ converges to $f$ in $L^{1}(\mu)$.

The weak-star sequential compactness of the dual closed unit ball allows us to assume that the sequence $\left(x_{n}^{*}\right)$ converges to some $x^{*}$ in the weak-star topology.

From Theorem 1 , for any $C \in \Sigma_{A}^{+}$,

$$
\left\langle\nu(C), x^{*}\right\rangle=\lim \left\langle\nu(C), x_{n}^{*}\right\rangle
$$


and, on the other hand,

$$
\lim \left\langle\frac{\nu(C)}{\mu(C)}, x_{n}^{*}\right\rangle=\lim \frac{1}{\mu(C)} \int_{C} f_{n} d \mu
$$

hence

$$
\left\langle\frac{\nu(C)}{\mu(C)}, x^{*}\right\rangle \geq \varepsilon / 2
$$

and so $x^{*} \geq \varepsilon / 2$ on $\mathscr{A}_{\nu}(A)$, despite the fact $\left\langle x^{*}, x\right\rangle \leq 0$.

Remark 1. The Banach space $\mathscr{C}\left(\left[0, \omega_{1}\right]\right)$, where $\omega_{1}$ is the first uncountable ordinal, has weak-star sequentially compact dual closed unit ball, whereas the functional $\lambda \rightarrow \lambda\left(\left\{\omega_{1}\right\}\right)$ is weak-star sequentially continuous on its dual space, and cannot be represented as a member of $\mathscr{C}\left(\left[0, \omega_{1}\right]\right)$.

So Theorem 1 does not include Theorem 2.

Remark 2. The proof of Theorem 2 can be changed to obtain the following result.

Let $X^{\#}$ be the subspace of $X^{* *}$ which consists of all those $x^{* *}$ satisfying the following property: for every bounded $\left(x_{n}^{*}\right)$ and every weak-star cluster point $x^{*}$ of $\left(x_{n}^{*}\right),\left\langle x^{* *}, x^{*}\right\rangle$ is a cluster point of $\left(\left\langle x^{* *}, x_{n}^{*}\right\rangle\right)$.

Then the range of $\nu$ is contained in $X$ provided that it is contained in $X^{\#}$ and $\nu$ satisfies the condition of Geitz.

Moreover, in this case Geitz's condition can be weakened as in [7]: $\operatorname{core}_{\nu}(A) \cap$ $\tilde{X} \neq \varnothing$ for any $A \in \Sigma^{+}$, where $\tilde{X}$ is the subspace of $X^{* *}$ which consists of those $x^{* *}$ which are weak-star cluster points of countable subsets of $X$. Indeed, to see that the core of the set $A$ in the proof of Theorem 2 does not meet $\tilde{X}$, we notice first that, for any given weak-star cluster point $x^{* *}$ of some sequence $\left(x_{n}\right)$, we can choose $\left(x_{n}^{*}\right)$ with $\left\|x_{n}^{*}\right\| \leq 1,\left|\left\langle x_{n}^{*}, x_{k}\right\rangle\right| \leq n^{-1}$ if $k \leq n$, and such that the sequence of densities $\left(f_{n}\right)$ converges to $f$ in $L^{1}(\mu)$. Then we obtain that $\left\langle x^{* *}, x^{*}\right\rangle=0$ although $x^{*} \geq \varepsilon / 2$ on $\mathscr{A}_{\nu}(A)$, if $x^{*}$ is a weak-star cluster point of $\left(x_{n}^{*}\right)$.

It is worthwhile to make some comments on the subspace $X^{\#}$.

(i) It is a closed subspace of $X^{* *}$ which contains the Hewitt realcompactification of $X$ (this follows from Corson's theorem [1]). For any nonrealcompact Banach space $X, X^{\#}$ does not coincide either with $X$ or with $X^{* *}$, as a consequence of James's theorem [2].

(ii) When $X$ has weak-star sequentially compact dual unit ball, weak-star sequentially continuous functionals on $X^{*}$ belong to $X^{\#}$, hence Theorem 2 follows from this Remark and Theorem 1. Nevertheless, we do not know if weak Geitz's condition is enough in Theorem 1 and so in Theorem 2.

(iii) $X^{\#} \cap \tilde{X}=X$. For, given a countable $Y \subset X$ and $x^{* *}$ in the weak-star closure of $Y$, we define $f: X^{*} \rightarrow \mathbb{R}$ by

$$
f\left(x^{*}\right)=\sum_{1}^{\infty} 2^{-n}\left|\left\langle x^{*}, x_{n}\right\rangle\right|
$$


where $\left(x_{n}\right)$ is an enumeration of $Y$. Then, if $x^{* *}$ were not in $X$, we could take $\left(x_{k}^{*}\right)$ in the unit ball of $X$ and $\delta>0$ so that $f\left(x_{k}^{*}\right)<k^{-1}$ but $\left|\left\langle x^{* *}, x_{k}^{*}\right\rangle\right| \geq$ $\delta$. If $x^{* *} \in X^{\#}$, then for some weak-star cluster point $x^{*}$ of $\left(x_{k}^{*}\right)$ we have $\left\langle x^{* *}, x^{*}\right\rangle=0$, which is a contradiction.

Remark 3. Modifying the proof of Theorem 2 we can prove Talagrand's theorem solving question (b) [8]. To see this we just need to extract one more subsequence from $\left(x_{n}^{*}\right)$ so that $f$ is the almost everywhere limit of $\left(f_{n}\right)$. This proof is similar to that given in [6].

We finally show that a pathological measure with values in the bidual of $l^{\infty}$ could be constructed from any pathological measure. By a pathological measure we mean here a vector measure which satisfies the assumptions of Theorem 1 but takes some of its values outside $X$.

Theorem 3. Let $\nu$ be an $X^{* *}$-valued vector measure, absolutely continuous with respect to the probability $\mu$. If $\nu$ satisfies Geitz's condition but $\nu(\Sigma)$ is not contained in $X$, then there is a vector measure $\eta: \Sigma \rightarrow\left(l^{\infty}\right)^{* *}$, which also satisfies Geitz's condition and whose range is not in $l^{\infty}$.

Proof. We proceed just as in Theorem 2, and choose $\varepsilon>0, f$ in the weak closure of the sets $\mathscr{B}_{Y}$ for any finite $Y \subset X, A \in \Sigma$ such that $f \geq \varepsilon / 2$ almost everywhere on $A$.

We choose $x \in X \cap \operatorname{core}_{\nu}(A)$ and $\left(x_{n}^{*}\right)$ in the unit ball of $X^{*}$ such that $\left\langle x_{n}^{*}, x\right\rangle \leq n^{-1}$ and $f_{n} \rightarrow f$ in $L^{1}(\mu)$, if $f_{n}$ is the density of $x_{n}^{*} \circ \nu$.

Let $T: X \rightarrow l^{\infty}$ be the bounded linear operator given by $T_{y}=\left(\left\langle x_{n}^{*}, y\right\rangle\right)$ for $y \in X$.

It is easy to check that the equation $\langle\eta(C), \lambda\rangle=\left\langle\nu(C), T^{*} \lambda\right\rangle$ for $\lambda \in\left(l^{\infty}\right)^{*}$, defines an absolutely continuous vector measure $\eta: \Sigma \rightarrow\left(l^{\infty}\right)^{* *}$.

Furthermore, this vector measure satisfies Geitz's condition. For, if $C \in \Sigma^{+}$, we take $y \in X \cap \operatorname{core}_{\nu}(C)$, and we have $T_{y} \in \operatorname{core}_{\eta}(C)$.

Finally, let us suppose that the range of $\eta$ lay in $l^{\infty}$. Then we fix a nonprincipal ultrafilter on the positive integers and we denote by $\lambda$ the member of the dual of $l^{\infty}$ induced by it.

If $\left(e_{n}\right)$ is the unit vector basis of $l^{1}$ regarded as members of $l^{\infty}$, then we have $T^{*} e_{n}=x_{n}^{*}$.

Let $x^{*^{n}}=T^{*} \lambda$. Since $\lambda$ is a weak-star cluster point of $\left(e_{n}\right)$ and $\eta(C)$ is weak-star continuous, $\langle\eta(C), \lambda\rangle=\left\langle\nu(C), x^{*}\right\rangle$ is a cluster point of the scalar sequence $\left(\left\langle\nu(C), x_{n}^{*}\right\rangle\right)$.

Moreover, as $T^{*}$ is weak-star to weak-star continuous, $x^{*}$ is a weak-star cluster point of $\left(x_{n}^{*}\right)$. It follows that $\left\langle x^{*}, x\right\rangle \leq 0$.

On the other hand, the average of $\nu$ on each $C \in \Sigma_{A}^{+}$satisfies

$$
\left\langle\frac{\nu(C)}{\mu(C)}, x^{*}\right\rangle \geq \varepsilon / 2
$$

contradicting the fact that $x \in \operatorname{core}_{\nu}(A)$. 


\section{REFERENCES}

1. H. Corson, The weak topology of a Banach space, Trans. Amer. Math. Soc. 101 (1961), 1-15.

2. J. Diestel, Geometry of Banach spaces-selected topics, Lecture Notes in Mathematics, Vol. 485, Springer-Verlag, Berlin-Heidelberg-New York, 1974.

3. J. Diestel and J. J. Uhl, Vector measures, Math. Surveys 15, Amer. Math. Soc., Providence, R.I., 1977.

4. N. Dunford and J. T. Schwartz, Linear operators. I: General theory, Interscience, New York, 1967.

5. R. F. Geitz, Geometry and the Pettis integral, Transl. Amer. Math. Soc. 269 (1982), 535-548.

6. R. Huff, Remarks on Pettis integrability, Proc. Amer. Math. Soc. 96 (1986), 402-404.

7. D. Sentilles and R. F. Wheeler, Pettis integration via the Stonian transform, Pacific J. Math. 107 (1983), 473-496.

8. M. Talagrand, Pettis integral and measure theory, Mem. Amer. Math. Soc., Providence, R.I., 1984.

Departamento de Análisis Matemático, Universidad de Sevilla, Aptdo. 1160, 41080Sevilla, Spain 